\title{
Global burden of prostate cancer: regional disparities in incidence, mortality, and survival
}

\author{
Miruna Dragomir ${ }^{1}$, Cécile Pizot ${ }^{1}$, Alina Macacu ${ }^{1}$, Alice Koechlin ${ }^{1,2}$, Maria Bota ${ }^{1,2}$, Peter Boyle ${ }^{1,2}$ \\ IInternational Prevention Research Institute (iPRI), Lyon, France \\ ${ }^{2}$ Strathclyde University Global Public Health Institute, Lyon, France
}

\begin{abstract}
Objectives: This article aims to provide a comprehensive overview of the worldwide burden of prostate cancer by exploring its global incidence, mortality, and survival.

Material and methods: Incidence rates were extracted from the CI5plus and NORDCAN databases. Prostate cancer deaths and populations were extracted from the WHO mortality database. For both incidence and mortality, age-standardised rates per 100,000 person-years (PY) were computed. Age-standardised relative survival was retrieved from the SEER database and the Eurocare-5, NORDCAN, and SURVCAN projects. For incidence and mortality, mean rates and annual percent changes (APC) over the last 10 years were computed.

Results: Recent incidence rates varied more than 25-fold across countries, ranging from 6.2 to 119 per $100,000 \mathrm{PY}$ in India and Brazil, respectively. Mortality rates also showed important disparities, ranging from 2.5 to 21.0 per 100,000 PY in Thailand and Estonia, respectively. Over the period 2003-2012 the incidence increased in 17 out of 26 countries. Over the period 2006-2015 the mortality decreased in 23 out of 30 countries. Age-standardised five-year survival from prostate cancer was very high and ranged from $34.8 \%$ in India to $90.0 \%$ in Finland. Survival trends in the USA and Northern Europe showed an increase starting in the 1970 s and reached almost $100 \%$ in recent years. Survival was poorer for distant stages (approximately 30\%), compared to localised and regional stages (almost 100\%).

Conclusions: Large variations in incidence and mortality were observed across countries, but survival rates remained high. Screening practices should be considered when interpreting these results because they largely influence incidence and survival.
\end{abstract}

KEY WORDS: prostate cancer, survival, incidence, mortality, burden, temporal trends, age-standardised rate.

ADDRESS FOR CORRESPONDENCE: Miruna Dragomir, International Prevention Research Institute (iPRI),

95 Cours Lafayette, 69006 Lyon, France, e-mail: miruna.dragomir@i-pri.org

\section{INTRODUCTION}

Prostate cancer is among the most commonly diagnosed cancers globally, and is the second most common cancer among men. In 2018, nearly 1.28 million men were estimated to be diagnosed with prostate cancer worldwide [1]. It was the most frequently diagnosed cancer in more than half of the world, mostly in America, Oceania, and Western Europe. Furthermore, prostate cancer was the fifth leading cause of death in men, with almost 359,000 estimated associated deaths in 2018 [1, 2]. Prostate-specific antigen (PSA) testing became commercially available from the middle to late 1980 s, and its use as a screening tool became widespread firstly in the USA, followed by other westernised countries such as Canada, Western European countries, and Australia [2]. Prostate-specific antigen screening detects prostate cancers early, but with high rates of overdiagnosis, identifying cancers that would not have developed or posed a threat to the patient during their lifetime.

Despite a myriad of epidemiological studies of this common cancer in Westernised countries, its aetiology remains relatively ambiguous. Established risk factors include age, race/ethnicity, and family history [3].

Understanding epidemiological trends might help identify and characterise risk factors and thus, provide further insight into prostate cancer prevention. 
The aim of this article was to describe a global picture of the worldwide burden of prostate cancer through its incidence, mortality, and survival, with a focus both on temporal trends and more recent figures.

\section{MATERIAL AND METHODS}

Prostate cancer was defined according to the International Classification of Disease (ICD, $7^{\text {th }}$ to $10^{\text {th }}$ revisions), using the following codes: A054 or 177 (ICD-7), A054 or 185 (ICD-8), B124 or 185 (ICD-9), and C61 or 1040 (ICD-10). Only malignant neoplasms were considered in this study. The period of study was 1953 through 2015, which corresponded to the longest period of available data.

\section{COUNTRY SELECTION}

The following countries, with a national cancer registry for both incidence and mortality, were selected for analysis: Bulgaria, Costa Rica, Croatia, Denmark, Estonia, Finland, Iceland, Lithuania, the Netherlands, New Zealand, Northern Ireland, Norway, Scotland, Slovakia, Slovenia, and Sweden. Some additional countries with regional registries for incidence were also selected: Australia, Brazil, Canada, Colombia, Japan, Thailand, the Republic of Korea, and the United States of America (USA). More details on the regional registries included can be found in Appendix 1 .

In order to give a global overview of the prostate cancer situation in the world, India and Uganda were added to the incidence analysis and Chile, Hong Kong, Israel, Mexico, Singapore, and South Africa were added to the mortality analysis. These additional countries for mortality were chosen according to the geographic location, the number of available years, and the quality of data [4]. Trends in prostate cancer incidence and mortality are presented by region as follows: Western Europe, Eastern Europe, Africa and the Middle East, Asia, Oceania, North America, and South and Central America.

\section{INCIDENCE DATA SOURCES}

Yearly age-standardised incidence rates were extracted from the Cancer Incidence in Five Continents database [5] for each country, except for the Northern European countries, for which data was extracted from the NORDCAN database [6].

\section{MORTALITY DATA SOURCES}

Pancreas cancer deaths registered in the World Health Organisation (WHO) [7] mortality database in November 2018 were extracted by country and five-year age group. Population data were also extracted from the WHO database and from the World Population Prospects of United Nations [8] when years were missing. When data were not available for one or two consecutive years, missing data were replaced by interpolations between previous and subsequent calendar years.

\section{SURVIVAL DATA SOURCES}

Several data sources were used to retrieve data on prostate cancer survival. The SEER database (Surveillance, Epidemiology, and End Results), which collects cancer related data from cancer registries across the USA, was explored with SEER ${ }^{\star}$ Stat software. Age-standardised relative survival data were extracted, for the period 1973 to 2015, by cancer stage, year of diagnosis, and survival duration. Stages at diagnosis were classified as: localised, regional, or distant tumours. The Eurocare- 5 project studies cancer survival in Europe and makes freely available online cancer survival data by cancer site, country, and survival duration, for the period 20002007 [9]. More detailed survival analyses were available for Nordic European countries through the NORDCAN project [6], specifically trends in prostate cancer survival between 1966 and 2018 .

A limited number of data were available for countries outside Europe and the USA, through the SURVCAN project [10], which provided survival data for Korea, Singapore, India, Thailand, and Hong Kong. Data was available for several cancer registries for each country, and an average survival among registries was extracted.

A literature search of the PubMed database was performed, in order to identify age-standardised five-year relative survival data for countries included in this study (more methodological details can be found in Appendix 2). Two publications were identified, reporting fiveyear age-standardised relative survival for Canadian non-aboriginal men, and for Singaporean men.

\section{STATISTICAL ANALYSIS}

For both incidence and mortality, age-standardised (ASR) rates were computed using the Segi world standard population as a reference and were reported for 100,000 person-years (PY). In Iceland, incidence and mortality rates fluctuated greatly over the period because of its small population size; therefore, a five-year smoothing average was used to represent the data.

In order to compare recent situations between different regions of the world, mean rates were computed for each country considering the period 2010-2012 for incidence and 2013-2015 for mortality. Also, annual percent changes (APC) over the last 10 available years (2003-2012 for incidence and 2006-2015 for mortality) were computed using a linear regression of the log-transformed rates.

For survival, only age-standardised relative survival values were extracted from each data source, for comparison purposes. Relative survival corresponds to the probability of surviving cancer in the absence of other causes of death and is defined as the ratio of the observed survival of patients to the survival expected in the general population (for the same gender, age, and calendar time). Since the underlying age structure of countries' populations is different, age-standardised relative survival is employed, generally using the International Cancer Survival Standards (ICSS) [11]. 

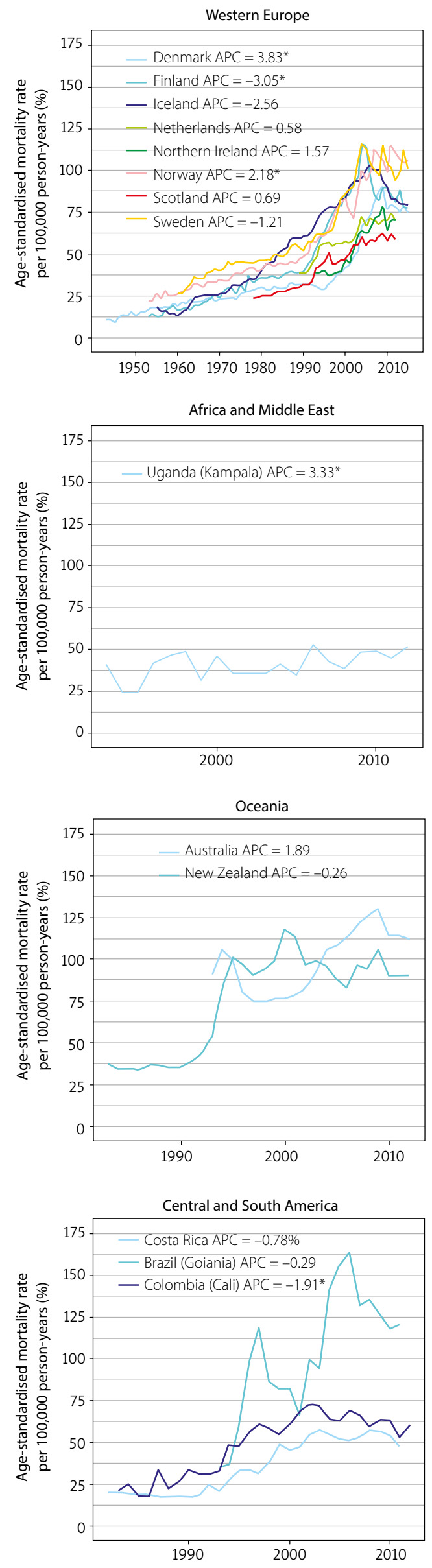
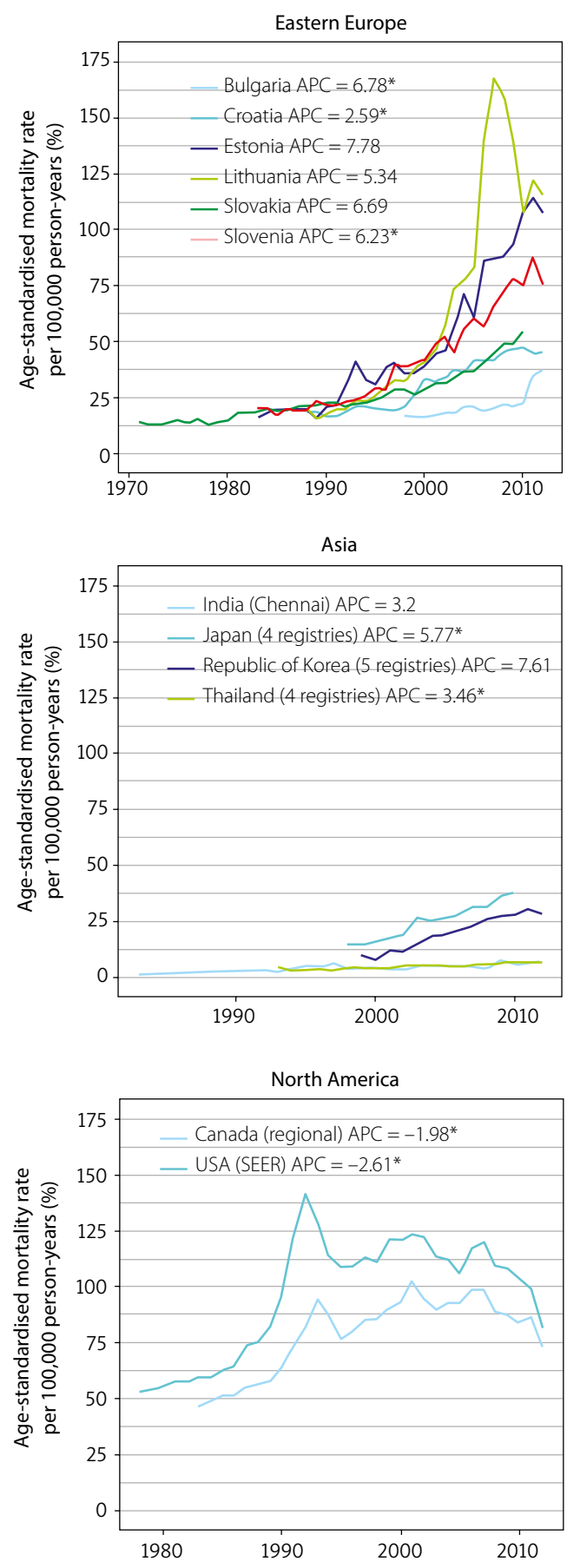

FIG. 1. Temporal trends in prostate cancer incidence, by region (APC - annual percent change computed for the last 10 available years [2003-2012]) 


\section{RESULTS}

\section{INCIDENCE}

Temporal trends in prostate cancer incidence are presented by region in Figure 1.

\section{Western Europe}

In Western European countries, the incidence of prostate cancer seemed to steadily increase over the period 1955-1990, followed by steeper increases in rates over the period 1990-2000 (Fig. 1).

Over the period 2003-2012, an upward trend in incidence rates was observed in some of the countries, with APCs of $+1.6 \%$ in Northern Ireland, $+2.2 \%$ in Norway, and $3.8 \%$ in Denmark, while in Finland, Iceland, and Sweden, a downward trend in incidence rates was observed, with APCs of $3.1 \%,-2.6 \%$, and $-1.2 \%$, respectively. On the other hand, incidence rates seemed to stabilise in the Netherlands and Scotland, with APCs of $+0.58 \%$ and $+0.69 \%$ over the $2003-2012$ period, respectively.

\section{Eastern Europe}

In most Eastern European countries, prostate cancer incidence rates have been steadily increasing starting from the mid-90s (Fig. 1). Over the period 2003-2012, a general upward trend was observed in all countries, with APCs ranging from $+2.6 \%$ (Croatia) to $+7.8 \%$ (Estonia), and statistically significant increases in Bulgaria, Croatia, and Slovenia.

In Lithuania, a peak in incidence rates was observed in 2007 (168 per 100,000 PY). This extraordinary increase was almost certainly the result of the introduction of the PSA testing in Lithuania in 2006 [12]. Following this peak, a decline in incidence rates was observed.

\section{Africa and Middle East}

Data on prostate cancer incidence from African and Middle Eastern countries remain generally scarce; only regional data were available for Uganda over the period 1993-2012 (Fig. 1). Incidence rates remained moderately low when compared to developed/developing countries, with estimated rates between 24.3 and 52.8 per 100,000 PY over the 1993-2012 period.

Over the period 2003-2012, an increase in prostate cancer incidence rates could be observed, with a corresponding APC of $+3.3 \%$.

\section{Asia}

Patterns of prostate cancer incidence in Asia can be classified in two groups: one enclosing Japan and the Republic of Korea, and one enclosing India and Thailand. Incidence rates in the latter group were lower and seemed to vary less than those in the former group, with ASRs remaining below 7.0 per 100,000 PY (Fig. 1). Over the 2003-2012 period, an upward trend was observed in both countries from this group, with APCs of $+3.2 \%$ and $+3.5 \%$ in India and Thailand, respectively.

In Japan and the Republic of Korea, the increase in rates was more pronounced over the aforementioned period, with APCs of $+5.8 \%$ and $+7.6 \%$, respectively.

\section{Oceania}

In Oceania, incidence rates were comparable to those in Western European and North American countries (Fig. 1). In New Zealand, where data was available for a longer period (1983-2012), a steep increase could be observed starting in the 1990s, similarly to Western Europe and North America.

Over the period 2003-2012, a stabilisation in incidence rates could be observed for New Zealand, while a slight upward trend could be observed in Australia, with corresponding APCs of $-0.26 \%$ and $1.89 \%$, respectively.

\section{North America}

In both Canada and the USA, prostate cancer incidence rates steadily rose up to the mid-90s, and more precisely to 1992 and 1993, respectively, where peaks in incidence can be observed, with estimated rates of 141.0 and 106.7 per 100,000 PY (Fig. 1).

Starting with the late 1990s, a significant decline in rates could be observed, with corresponding APCs of $-1.98 \%$ and $-2.61 \%$ over the period $2003-2012$ for Canada and the USA, respectively.

\section{Central and South America}

Patterns of prostate cancer incidence were very similar in Colombia and Costa Rica, with slightly higher rates in Colombia (Fig. 1), but with ASRs remaining below 75 per 100,000 PY in both countries. Over the period 2003-2012, a decline in prostate cancer was observed in both countries, with corresponding APCs of $-1.91 \%$ and $-0.78 \%$ in Colombia and Costa Rica, respectively.

Despite no active screening in Brazil [13], the highest rates were observed there, with peaks in 1997 (111.1 per 100,000 PY) and 2006 (186.7 per 100,000 PY) and with an estimated APC of $-0.29 \%$ over the period 2003-2012.

\section{Recent rates}

Over the period 2010-2012, prostate cancer incidence rates varied more than 25 -fold across countries, between 6.2 per $100,000 \mathrm{PY}$ in India and 119.0 per 100,000 PY in Brazil (Fig. 2).

The highest incidence rates were observed in both Western and Eastern Europe and North America, as well in Oceania and some of the Central and South American countries, with rates of 119.0 per 100,000 PY in Brazil followed by Lithuania (115.2 per 100,000 PY) and Australia (114.1 per 100,000 PY). Inversely, the lowest incidence rates were observed in Asian countries, with mean rates of 6.2 and 6.8 per 100,000 PY in India and Thailand, respectively. 


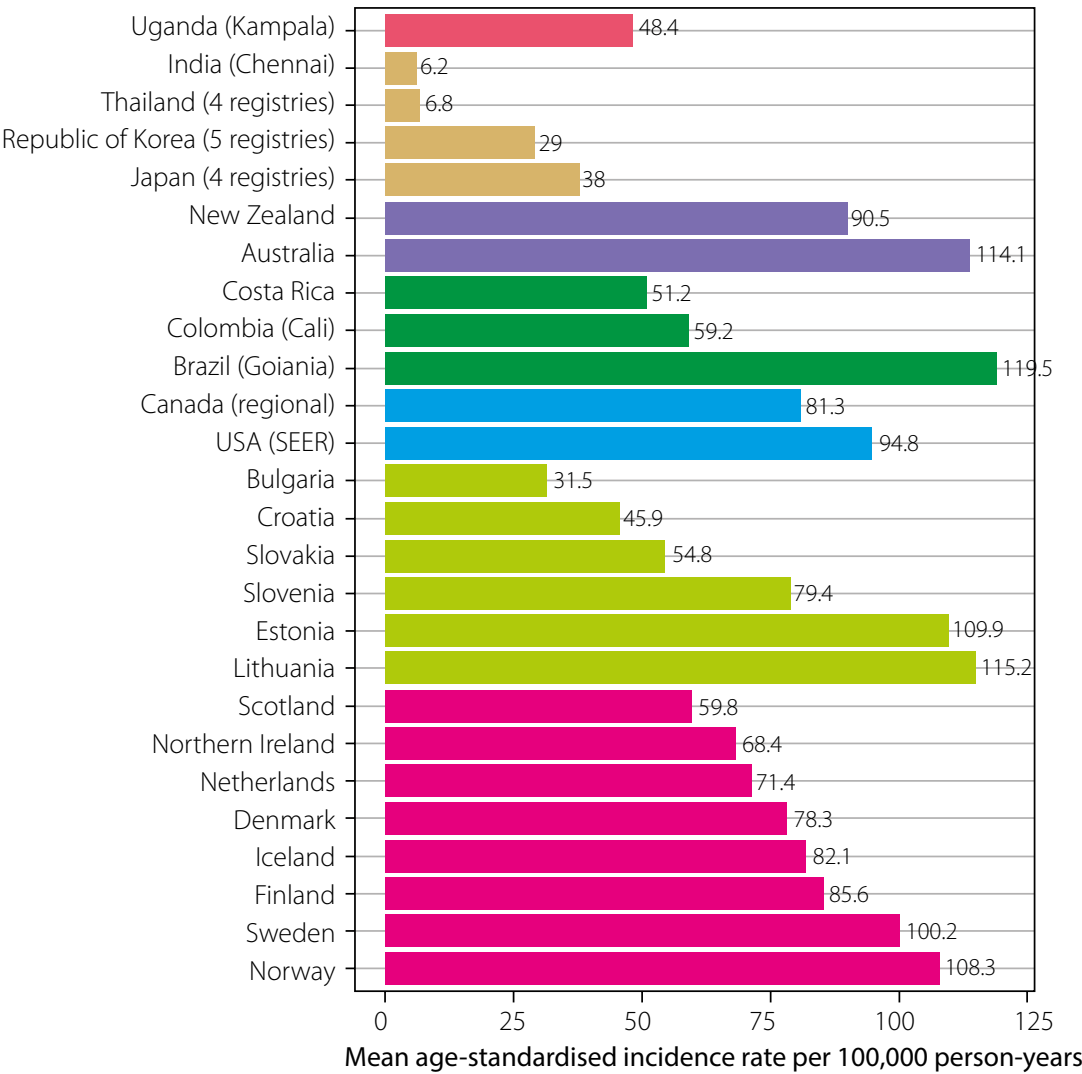

(2010-2012)

Africa and the Middle East

- Central and South America
North America

- Western Europe
Asia
Eastern Europe
Oceania

FIG. 2. Mean age-standardised prostate cancer incidence over the period 2010-2012, by country

\section{MORTALITY}

Temporal trends in prostate cancer mortality are presented by region in Figure 3.

\section{Western Europe}

Trends in prostate cancer mortality rates were similar across all Western European countries over the studied period, with steep increases from the 1950s, reaching a peak around 1995, followed by steep decreases (Fig. 3). More recently (2006-2015), a downward trend could be observed in all countries, with APCs ranging from $-2.63 \%$ (the Netherlands) to $-0.25 \%$ (Scotland). The decline was significant in Denmark, Finland, Netherlands, Norway, and Sweden.

Presently, prostate cancer mortality rates are higher than in the 1950s, but recent trends show important declines, with this tendency probably persisting in the near future.

\section{Eastern Europe}

Since the 1980s, steep increases in mortality were observed in most Eastern European countries until the mid-2000s, followed by a stabilisation of the rates in Croatia (APC over the 2006-2015 period of $+0.03 \%$ ) and Estonia (APC over the 2006-2015 period of $+0.9 \%$ ), while decreasing trends were noticed in Lithuania (APC over the 2006-2015 period of $-1.9 \%$ ) and Slovenia (APC over the 2006-2015 period of $-2.9 \%$ ) (Fig. 3).

The mortality trend in Slovakia was slightly different: an increase in mortality was observed until 2000, followed by a decline in 2008, which was followed by a new increase in mortality rates (APC over the 20062013 period of $+2.3 \%$ ). Mortality rates in Bulgaria were lower compared to other Eastern European countries, and seemed to increase steadily since 1965 , with an APC of $+1.3 \%$ over the last 10 years.

\section{Africa and Middle East}

Coverage of death registration was very low in Africa and the Middle East; therefore, only two countries were selected: South Africa and Israel. Prostate cancer mortality rates were more than two-times higher in South Africa than in Israel over the period 1993-2015 (Fig. 3).

In South Africa, prostate cancer mortality rates were generally stable over the studied period. More recently (2006-2015), a slight upward trend in rates was observed, with an APC of $+1.2 \%$ over this period. 

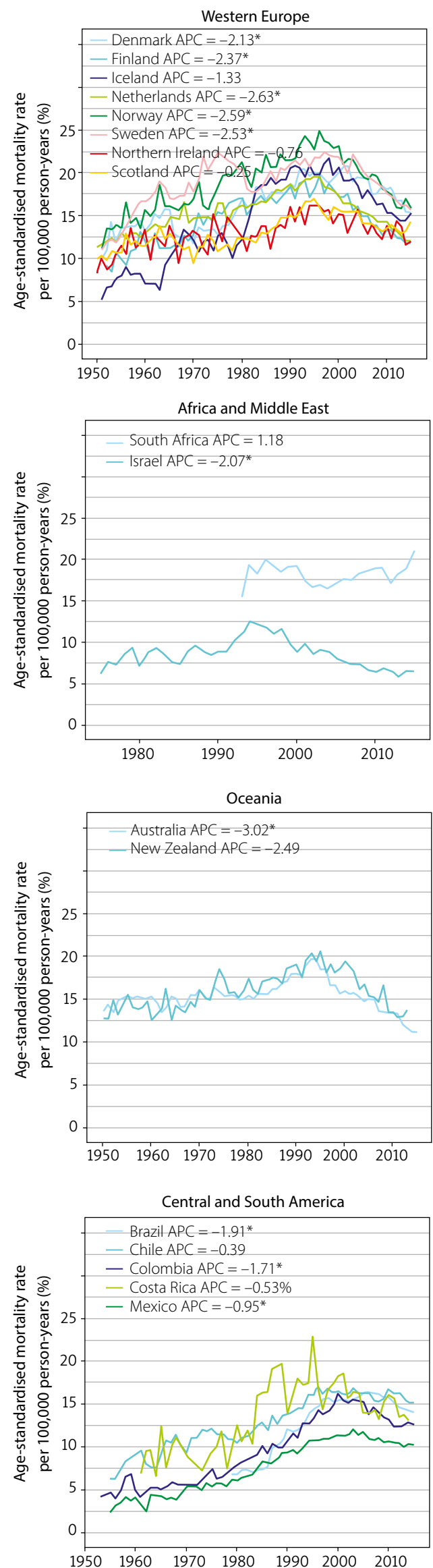
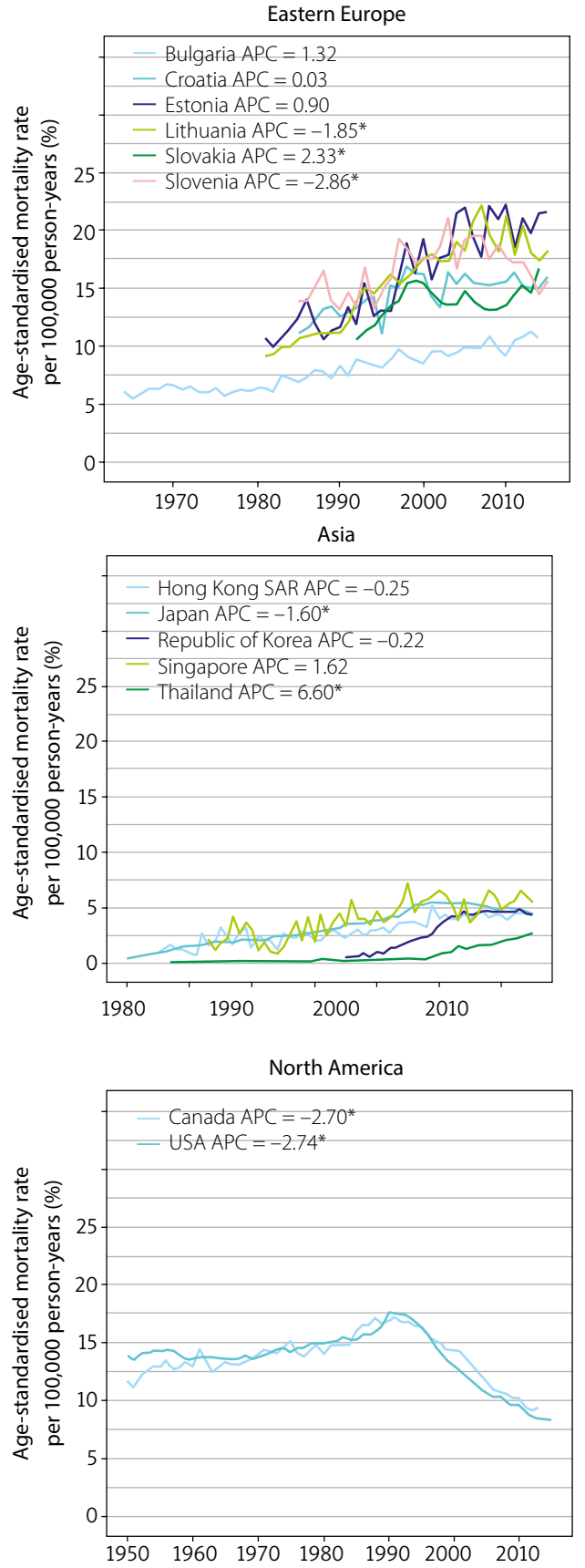

FIG. 3. Temporal trends in prostate cancer mortality, by region (APC - annual percent change computed for the last 10 available years [2006-2015]) 
Regarding Israel, the longer period of time allowed the identification of the same tendency as in Western Europe, North America, and Oceania, i.e. a steady raise in prostate cancer mortality (from 6.2 per 100,000 PY in 1975 to 12.4 per 100,000 PY in 1994) followed by a decline in mortality rates. This was confirmed by more recent figures: over the period 2006-2015, a significant decline in mortality rates was observed, with a corresponding APC of $-2.1 \%$.

\section{Asia}

Compared to all other regions, lower prostate cancer mortality rates were observed in Asian countries over the period 1950-2015 (Fig. 3), with rates lower than 2.0 in the 1950s and lower than 6.5 per 100,000 PY for the most recent years (2013-2015) (Fig. 4). This is due to the fact that in this region, incidence rates were lower than in other regions.

Over the period 2006-2015, there was a significant upward trend in rates in Thailand, while a significant downward trend was observed in Japan, with corresponding APCs of $+6.6 \%$ and $-1.6 \%$, respectively. In all the other countries, rates seemed more stable over the same period with APCs of $-0.3 \%,-0.22 \%$, and $1.62 \%$ in Hong Kong, the Republic of Korea, and Singapore, respectively.

\section{Oceania}

Prostate cancer mortality rates were similar in Australia and New Zealand throughout the studied period (Fig. 3). Similarly to Western Europe and North America, a mortality peak was clearly observed in the mid1990s. This peak was firstly observed in Australia with a mortality rate of 19.6 per 100,000 PY in 1993 and then in New Zealand, in 1995, when prostate cancer mortality peaked at 20.6 per $100,000 \mathrm{PY}$.

Over the studied period, steady increases in mortality rates were observed from 1950 up to 19931995, followed by steep decreases in both countries. This downward trend was confirmed by more recent figures, with APCs of $-3.0 \%$ in Australia and $-2.5 \%$ in New Zealand, over the period 2006-2015. Due to this important decrease in mortality, rates are presently similar to those in the 1950s, and there is no sign that this decreasing trend will stop in the near future.

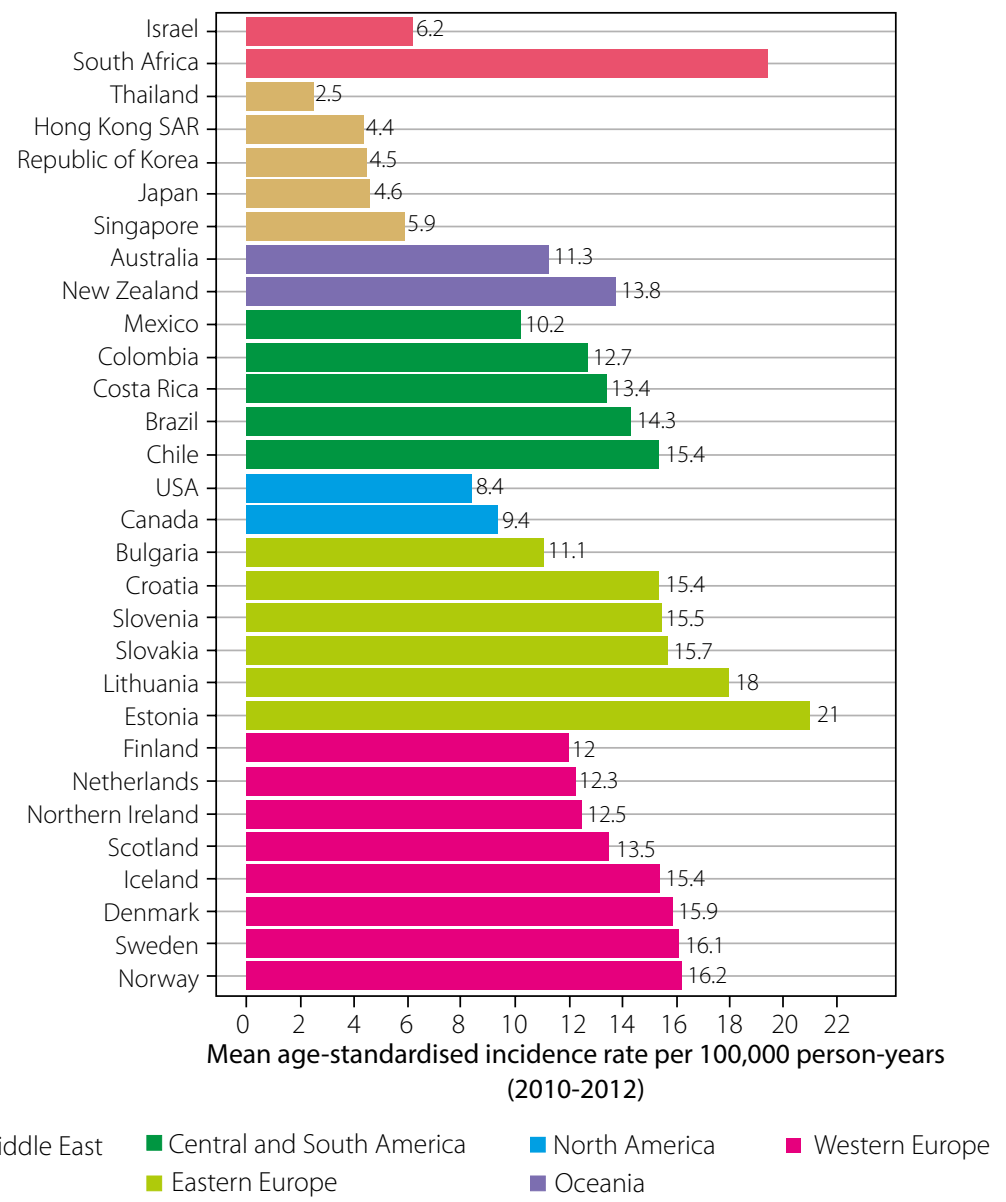

FIG. 4. Mean age-standardised prostate cancer mortality over the period 2013-2015, by country 
A

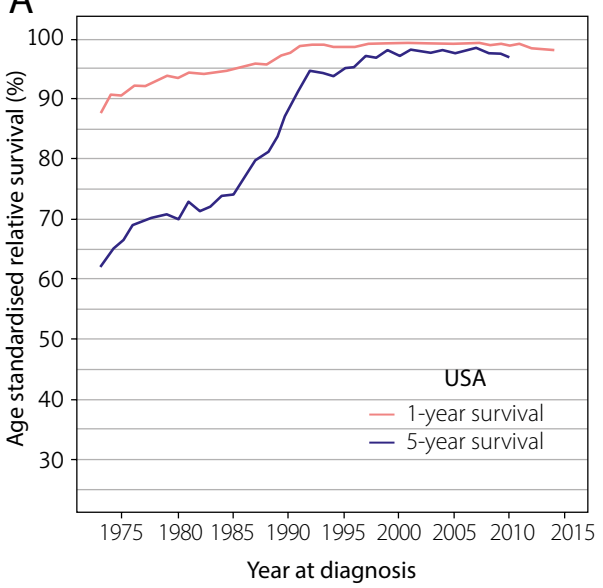

C

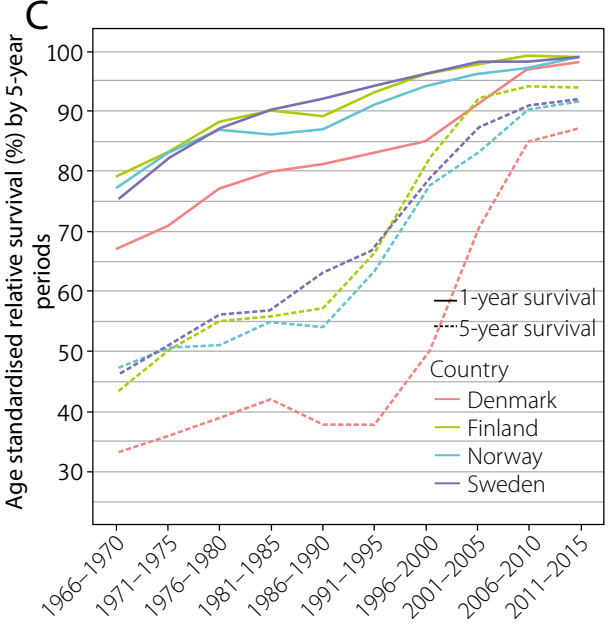

Period of diagnosis
B

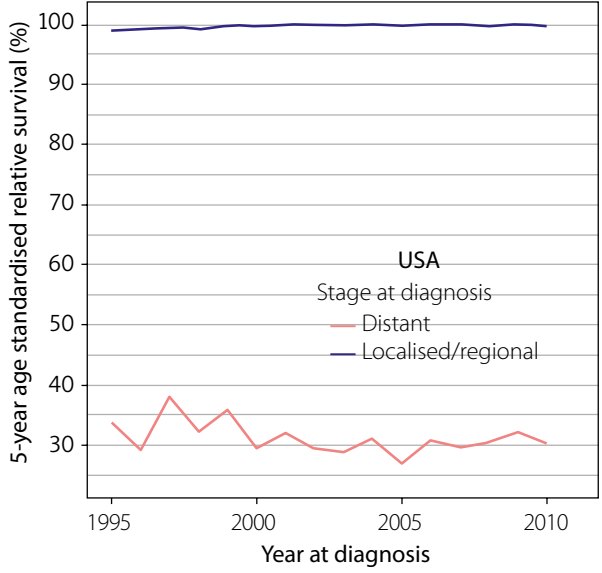

D

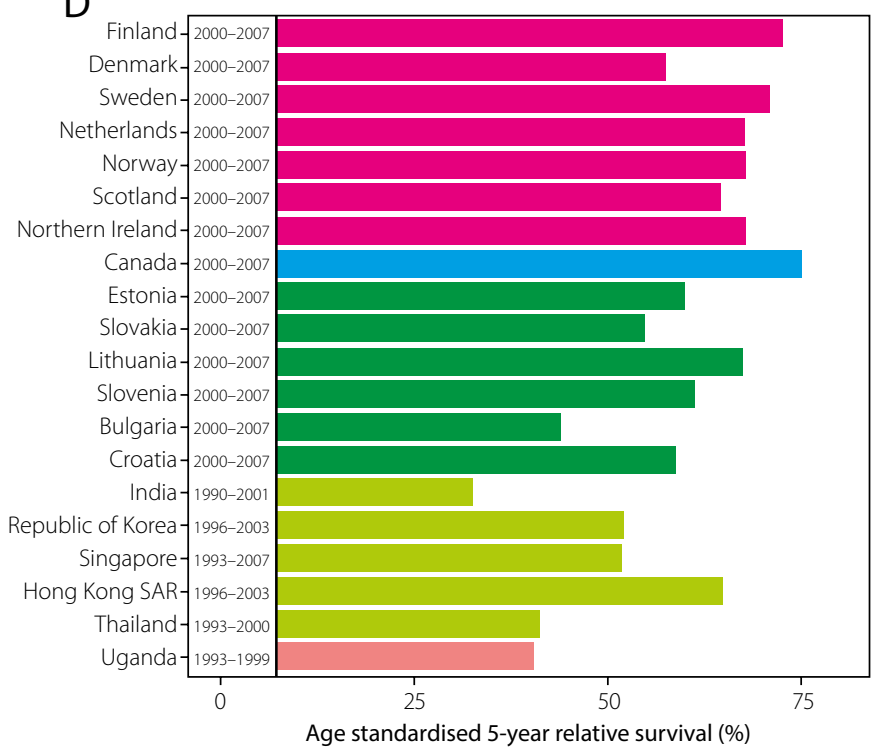

$\square$ Africa and the Middle East Asia
Eastern Europe

North America

FIG. 5. A) USA - SEER data. One- and five-year age-standardised relative survival by year at diagnosis; B) USA - SEER data. Five-year age-standardised relative survival by stage at diagnosis and year at diagnosis; C) Nordic European countries - NORDCAN data. One- and five-year age-standardised relative survival by country and period of diagnosis; D) Five-year age-standardised relative survival by country. Data sources: 1) European countries - Eurocare-5; 2) Asian and African countries: SURVCAN; 3) North America country - [1]. Eurocare-5 data are calculated for the 2000-2007 period, while other countries each have survival calculated for different time periods [14]

\section{North America}

Prostate cancer mortality rates were similar in the USA and Canada over the studied period (Fig. 3). Similarly to the situation in Western European countries, a mortality peak was clearly observed in the early 1990s. In both countries, the peak was observed in 1991, with mortality rates of 17.5 and 17.3 per $100,000 \mathrm{PY}$ in the USA and Canada, respectively.

In both countries, steady increases in mortality were observed from 1950 to 1991, followed by steep decreases. More recently (2006-2015), significant decreases in mortality rates were observed in both countries, with corresponding APCs of $-2.70 \%$ and $-2.74 \%$ for Canada and the USA, respectively. Nowadays, due to the important decrease in mortality since 1991, prostate cancer mortality rates are lower than in the 1950s, and there is no sign that this decreasing trend will stop in the near future.

\section{Central and South America}

Prostate cancer mortality rates were similar in all selected countries of Central and South America over the studied period (Fig. 3). Since the 1960s (1970s for Brazil), steep increases in mortality rates were observed in all countries until 1995 for Costa Rica and Mexico, 1996 for Chile, 2000 for Colombia, and 2006 for Brazil. 
At these inflection points, the highest mortality rate was observed in Costa Rica (22.9 per 100,000 PY), while the lowest mortality rate was observed in Mexico (12.9 per 100,000 PY). Following these peaks, declines in prostate cancer mortality rates were observed in all Central and South American countries.

More recently (2006-2015), a general downward trend in mortality rates was observed in all countries, with APCs ranging from $-1.7 \%$ (Colombia) to $-0.39 \%$ (Chile).

\section{Recent rates}

Over the period 2013-2015, important disparities were observed across countries, with prostate cancer mortality rates ranging from 2.5 per $100,000 \mathrm{PY}$ in Thailand to 21.0 per 100,000 PY in Estonia (Fig. 4). The highest mortality rates were observed in Eastern Europe, with rates of 21.0 per $100,000 \mathrm{PY}$ in Estonia and 18.0 per 100,000 PY in Lithuania, followed by Western Europe, with rates of 16.2 and 16.1 per 100,000 PY in Norway and Sweden, respectively. Conversely, the lowest mortality rates were observed in Asian countries with rates of 2.5 per 100,000 PY in Thailand, 4.4 per 100,000 PY in Hong Kong, 4.6 per 100,000 PY in the Republic of Korea, 4.6 per 100,000 PY in Japan, and 5.9 per 100,000 PY in Singapore.

\section{SURVIVAL}

Survival of prostate cancer is very high. In the USA, one-year age-standardised relative survival increased from $87.5 \%$ in 1973 to $98.0 \%$ in 2014 , while five-year survival increased from $62.0 \%$ in 1973 to $97.0 \%$ in 2010 (Fig. 5A).

Stage at diagnosis in the SEER database for prostate cancer is classified as localised/regional or distant. The equivalent in the AJCC (American Joint Committee on Cancer) staging would be: stages I and II for localised tumours, stages III and IV that have not spread far for regional tumours, and cancers that have spread to distant areas for distant tumours [15]. Prostate cancer diagnosed at distant stages has poorer five-year relative survival (approximately 30\%); however, almost all prostate cancer patients diagnosed at localised and regional stages survive five years after diagnosis (Fig. 5B). The stage-specific survival of prostate cancer has remained stable since 1995. Since the introduction of PSA screening in the USA in the early 1990s, most prostate cancers have been diagnosed in the early stages: $80 \%$ with localised disease, $12 \%$ with regional disease, $4 \%$ with distant disease, and $3 \%$ unstaged [15]. This corresponds with the sharp increase in five-year survival observed in Figure 5A.

Age-standardised survival trends in Nordic European countries showed that five-year relative survival increased from $35-45 \%$ in $1966-1970$ to $87-94 \%$ in 2011 2015. Denmark had the lowest survival rates, and Sweden the highest (Fig. 5C).

Overall, Western European Countries had similar five-year age-standardised relative survival for the period 2000-2007 (69.2-90.0\%). Eastern European countries had more modest five-year relative survival (50.5-82.8\%) (Fig. 5D). Regional data available for other countries from Asia and Africa [10] showed lower five-year age-standardised survival, ranging between $34.8 \%$ for India and 79.3\% for Hong Kong SAR (Fig. 5D). One article identified through the literature search [16] reported five-year age-standardised survival for Singapore by diagnosis year between 1970 and 2000; the authors found that prostate cancer survival varied little during this time period, between $50 \%$ and $60 \%$.

\section{DISCUSSION}

This article presents the worldwide burden of prostate cancer through its incidence, mortality, and survival, focusing on both recent rates and temporal trends.

Globally, large variations in recent prostate cancer incidence and mortality rates were highlighted in this article. When comparing rates in recent years, the incidence varied more than 25 -fold and mortality around 10 -fold among the included countries. Disparities in current PSA testing practices among countries heavily influenced recent incidence rates because PSA testing is more widespread in more developed than in less developed countries [17-19]. There was no clear geographic or economic explanation for the disparities in recent mortality rates. Indeed, in the list of the 10 highest mortality rates, Eastern European countries stand alongside Western European countries, South Africa, and Chile, while in the 10 lowest mortality rates, Asian countries stand alongside Israel, North American countries, and Mexico. The lowest mortality rates were observed in Asian countries.

Although Canada and the USA were among countries with the highest incidence, they were also among those with the lowest mortality; this could be explained by the high prevalence of PSA testing and the widespread access to healthcare and effective treatments.

Dramatic increases in incidence rates since the 1990s in most high-income countries (Oceania, North Ameri$\mathrm{ca}$, and Western Europe) were probably due to the intensive use of the PSA testing for early detection and diagnosis $[20,21]$. The decrease in incidence following these trends can be explained by the early detection through screening of some cancers that would have been detected in the future [22]. On the other hand, incidence rates still on the rise were observed in developing countries (e.g. countries from Eastern Europe, Asia) in which the use of PSA testing became more widespread later on $[18,23]$.

Taking into consideration the large influence of screening practices on the prostate cancer incidence trends, their interpretation should be done with caution. Perhaps mortality trends rather than incidence might better reflect the actual risk across different populations [24], but they can still be affected by the cancer diagnosis and death certification processes.

Important declines in mortality observed since the 1990s in most high-income countries may be explained 
by the improvements in radiation therapy $[25,26]$ and surgical techniques $[27,28]$, especially for early-stage prostate cancer mainly detected by PSA testing since the 1990s. The less pronounced decreases in prostate cancer mortality observed in Eastern Europe and in Latin America would be explained by the less widespread use of PSA testing and limited access to efficient treatments $[18,29]$. Regarding Asia, the westernisation of lifestyle could be one explanation for the observed mortality trends $[18,30]$. Moreover, incidence in Asian countries appears to be increasing, which would result in more prostate cancer cases, but with limited access to effective treatment and surgical advances, which would explain the increase in mortality.

Survival of prostate cancer patients was very high, with localised and regional prostate cancers in the US showing $97 \%$ relative five-year survival. Survival trends have also been increasing, synchronised with the increases in incidence after the introduction of PSA screening.

Incidence and five-year survival rates were found to be correlated, with Pearson's correlation coefficient $R=0.73$ [31]. Prostate cancer survival could be modified by early detection of the disease, which could make the survival times appear longer artificially (lead-time bias). The PSA testing implemented in a country should be kept in mind when analysing survival and survival trends.

Survival of prostate cancer patients showed differences among countries. Given that earlier detection of prostate cancer leads to better survival, countries with widespread screening programmes, better awareness of the disease among the population, and greater availability of resources generally have increased incidence and survival rates of prostate cancer. Survival rates for prostate cancer should be interpreted with care, in the context of incidence and mortality data, and screening practices.

Geographical location appeared to influence the burden of prostate cancer, but other factors such as age also play a role. Prostate cancer is primarily a disease of older age, with low incidence among people below 60 years old. The widespread use of PSA testing has also lowered the median age at diagnosis over the last three decades, with more and more younger men being diagnosed [22]. Mortality from prostate cancer increases with age, with an eight-fold increase in risk in men over 70 years old when compared to younger men (50-59 years old). In the USA, survival gradually increased with age for men between 55 and 85 years old. An analysis of prostate cancer in younger men found that overall survival in younger men was better than in older men with more comorbidities; however, disease-specific survival at 10 years was not significantly improved [32]. The authors also reported that for high-grade tumours, younger men had poorer survival.

Our study attempted to present a comprehensive overview of the epidemiology of prostate cancer. However, some limitations should be addressed. Firstly, for the incidence analysis, in addition of data from national cancer registries, regional cancer data were used in order to have worldwide representativeness. However, regional data could be inaccurate because they do not cover the whole population and the whole territory. For instance, regional rates could be underestimated due to lower precision for local/regional data when compared to national data. Moreover, when only one regional registry exists in an urbanised area of the country, data could also be underestimated for countries with large rural populations. Also, there are disparities in methods of registering and quality, coverage, and completeness of cancer registration across countries [33]. Secondly, for comparison purposes, an effort was made to include the same countries for the incidence and mortality analyses, which led to the inclusion of countries with low quality of mortality data (e.g. Thailand and South Africa) [4]. Thirdly, data for incidence was available only up until 2012, while data for mortality was more recent (up until 2015). Further updates would be necessary in order to also have more recent results for incidence. This would also ensure better comparability of incidence and mortality trends. Fourthly, for the survival analysis, the direct comparison between data sources was limited, due to the fact that Eurocare and SEER data used the ICSS, NORDCAN data used age-standardisation with age groups different from the ICSS, and Survcan data used an alternative approach to age-standardisation [10]. Finally, data for the African region remained scarce: data only for Uganda and South Africa were available for the incidence and mortality analyses, respectively. Thus, it makes difficult any conclusion on the burden of prostate cancer in Africa.

To conclude, there are worldwide disparities in incidence, mortality, and survival of prostate cancer. Additional studies should further investigate the reasons underlying these disparities by evaluating potential associations with socio-economic factors and/or screening practices.

\section{DISCLOSURE}

The authors report no conflict of interest.

\section{REFERENCES}

1. Ferlay J, Lam F, Colombet M, Mery L, Piñeros M, Znaor A, Soerjomataram I, Bray F. Global Cancer Observatory: Cancer Today. In. Lyon, France: Lyon, France: International Agency for Research on Cancer 2018. Available from: https://publications.iarc.fr/Databases/Iarc-Cancerbases/Cancer-Today-Powered-By-GLOBOCAN-2018--2018 (accessed: 30 October 2019).

2. Bray F, Ferlay J, Soerjomataram I, et al. Global cancer statistics 2018: GLOBOCAN estimates of incidence and mortality worldwide for 36 cancers in 185 countries. CA Cancer J Clin 2018; 68 (6): 394-424.

3. Patel AR, Klein EA. Risk factors for prostate cancer. Nat Clin Pract Urol 2009; 6 (2): 87-95. 
4. Mathers CD, Fat DM, Inoue $M$ et al. Counting the dead and what they died from: an assessment of the global status of cause of death data. Bull World Health Organ 2005; 83 (3): 171-177.

5. Ferlay J, Colombet M, Bray F. Cancer Incidence in Five Continents. CI5plus. IARC CancerBase No. 9. In. Lyon, France: International Agency for Research on Cancer 2018. Available from: https://ci5.iarc.fr/CI5I-X/old/vol10/CI5vol10.pdf (accessed: 30 October 2019).

6. Engholm G, Ferlay J, Christensen N, et al. NORDCAN: Cancer Incidence, Mortality, Prevalence and Survival in the Nordic Countries. Association of the Nordic Cancer Registries. In. Danish Cancer Society 2018. Available from: https: https://www-dep. iarc.fr/NORDCAN/english/frame.asp (accessed: 30 October 2019).

7. WHO Mortality database. Available from: https://www.who.int/ healthinfo/mortality_data/en/ (accessed: 2 November 2019).

8. United Nations. World Population Prospects. The 2015 Revision. In. New York, USA: United Nations 2015. Available from: https://population.un.org/wpp/publications/files/key_findings_wpp_2015.pdf (accessed: 2 November 2019).

9. Lepage C, Capocaccia R, Hackl M et al. Survival in patients with primary liver cancer, gallbladder and extrahepatic biliary tract cancer and pancreatic cancer in Europe 1999-2007: Results of EUROCARE-5. Eur J Cancer 2015; 51 (15): 2169-2178.

10. Sankaranarayanan R. Cancer survival in Africa, Asia, the Caribbean and Central America. Introduction. IARC Sci Publ 2011; (162): 1-5.

11. Corazziari I, Quinn M, Capocaccia R. Standard cancer patient population for age standardising survival ratios. Eur J Cancer 2004; 40 (15): 2307-2316.

12. Smailyte G, Aleknaviciene B. Incidence of prostate cancer in Lithuania after introduction of the Early Prostate Cancer Detection Programme. Public Health 2012; 126 (12): 1075-1077.

13. Busato WF Jr, Almeida GL. Prostate cancer screening in Brazil: should it be done or not? Int Braz J Urol 2016; 42 (6): 1069-1080.

14. Mazereeuw MV, Withrow DR, Nishri ED, et al. Cancer incidence and survival among Metis adults in Canada: results from the Canadian census follow-up cohort (1992-2009). CMAJ 2018; 190 (11): E320-E326.

15. Brawley OW. Prostate cancer epidemiology in the United States. World J Urol 2012; 30 (2): 195-200.

16. Chia SE, Tan CS, Lim GH, et al. Incidence, mortality and survival patterns of prostate cancer among residents in Singapore from 1968 to 2002. BMC Cancer 2008; 8: 368.

17. Baade PD, Yu XQ, Smith DP, et al. Geographic disparities in prostate cancer outcomes--review of international patterns. Asian Pac J Cancer Prev 2015; 16 (3): 1259-1275.

18. Center MM, Jemal A, Lortet-Tieulent J, et al. International variation in prostate cancer incidence and mortality rates. Eur Urol 2012; 61 (6): 1079-1092.

19. Jemal A, Center MM, DeSantis C, Ward EM. Global patterns of cancer incidence and mortality rates and trends. Cancer Epidemiol Biomarkers Prev 2010; 19 (8): 1893-1907.

20. Dickinson J, Shane A, Tonelli M, et al. Trends in prostate cancer incidence and mortality in Canada during the era of prostate-specific antigen screening. CMAJ Open 2016; 4 (1): E73-E79.

21. Etzioni R, Penson DF, Legler JM, et al. Overdiagnosis due to prostate-specific antigen screening: lessons from U.S. prostate cancer incidence trends. J Natl Cancer Inst 2002; 94 (13): 981-990.

22. Brawley OW. Trends in prostate cancer in the United States. J Natl Cancer Inst Monogr 2012; 2012 (45): 152-156.

23. Zhou CK, Check DP, Lortet-Tieulent J, et al. Prostate cancer incidence in 43 populations worldwide: An analysis of time trends overall and by age group. Int J Cancer 2016; 138 (6): 1388-1400.

24. Hsing AW, Tsao L, Devesa SS. International trends and patterns of prostate cancer incidence and mortality. Int J Cancer 2000; 85 (1): 60-67.

25. Peschel RE, Colberg JW. Surgery, brachytherapy, and external-beam radiotherapy for early prostate cancer. Lancet Oncol 2003; 4 (4): 233-241.

26. Jani AB, Hellman S. Early prostate cancer: clinical decision-making. Lancet 2003; 361 (9362): 1045-1053.

27. Walsh PC. Anatomic radical prostatectomy: evolution of the surgical technique. J Urol 1998; 160 (6 Pt 2): 2418-2424.

28. Langley SE, Laing R. Prostate brachytherapy has come of age: a review of the technique and results. BJU Int 2002; 89 (3): 241-249.

29. Bray F, Kiemeney LA. Epidemiology of Prostate Cancer in Europe: Patterns, Trends and Determinants. In: Management of Prostate Cancer: A Multidisciplinary Approach, Bolla M, van Poppel H (eds.). Cham: Springer International Publishing 2017: 1-27.

30. Kimura T, Egawa S. Epidemiology of prostate cancer in Asian countries. Int J Urol 2018; 25 (6): 524-531.

31. Trama A, Foschi R, Larranaga N, et al. Survival of male genital cancers (prostate, testis and penis) in Europe 1999-2007: Results from the EUROCARE-5 study. Eur J Cancer 2015; 51 (15): 2206-2216

32. Lin DW, Porter M, Montgomery B. Treatment and survival outcomes in young men diagnosed with prostate cancer: a Population-based Cohort Study. Cancer 2009; 115 (13): 2863-2871.

33. Bray F, Znaor A, Cueva P, et al. Planning and developing population-based cancer registration in low-and middle-income settings. In IARC Technical publication No 43. World Health Organisation, France 2015.

\section{AUTHORS' CONTRIBUTIONS}

$M D, P B$ prepared research concept and design of the publication. MD, CP, AM collected and analysed data. MD wrote the article. All authors contributed to the final version of publication. 
Appendix 1. List of regional registries included, in alphabetical order

\begin{tabular}{|l|l|l|}
\hline Country & \multicolumn{1}{|c|}{ Registries } & $\begin{array}{c}\text { Period } \\
\text { available }\end{array}$ \\
\hline Australia & Queensland, South Australia, Tasmania, Victoria, Western Australia, Northern territories & $1993-2012$ \\
\hline Brazil & Goiania & $1993-2012$ \\
\hline Canada & All except Nunavut, Quebec, and Yukon & $1983-2012$ \\
\hline Colombia & Cali & $1983-2012$ \\
\hline India & Chennai & $1983-2012$ \\
\hline Japan & Miyagi, Nagasaki, Osaka, Fukui & $1998-2010$ \\
\hline Korea & Busan, Seoul, Ulsan, Gwangju, Incheon & $1999-2012$ \\
\hline Thailand & Chiang Mai, Knon Kaen, Songkhla, Lampang & $1993-2012$ \\
\hline Uganda & Kampala & $1993-2012$ \\
\hline USA & $\begin{array}{l}\text { California: San Francisco; Connecticut; Georgia: Atlanta; Hawaii; lowa; Michigan: Detroit; New } \\
\text { Mexico; Utah; Washington: Seattle (SEER registries) }\end{array}$ & $1978-2012$ \\
\hline
\end{tabular}

\section{Appendix 2. Literature search for survival data}

Literature search of the PubMed database was performed in November 2018, for publications reporting national or regional prostate cancer survival rates. Eligible studies needed to be population-based and to report age-standardised five-year relative survival. Studies on specific sub-groups of populations (such as specific treatment) were excluded. Only reports for the countries included in this study (as described in the Methods section) were included.

The search strategy yielded 706 results, 637 of which were excluded based on title and abstract. Of the remaining 69 publications, only two fit the eligibility criteria and were included in this study. The following PubMed search query was used:

(survival[Title/Abstract])

AND

("prostate cancer"[Title/Abstract] OR “prostate neoplasm”[Title/Abstract] OR "prostate carcinoma”[Title/ Abstract] )

AND

(europe[Mesh] OR "north america"[Mesh] OR "south america"[Mesh] OR "central america"[Mesh] OR "asia”[Mesh] OR africa[Mesh] OR “oceania”[Mesh] OR countr*[Title/Abstract] OR nation*[Title/Abstract] OR world[Title/Abstract] OR global[Title/Abstract])

AND

("rate*"[Title/Abstract] OR "trend" ${ }^{\star ”[T i t l e / A b s t r a c t]) ~}$ 